\title{
Analysis and Optimum Design of a Portable Q-Switch Based on Raman-Nath Diffraction
}

\author{
Huadong Gu1,2, Zhongxing Shao', Jie Yang1,2, Chenqi Zheng, ${ }^{1,2}$, Ruitao Chen ${ }^{1,2}$ \\ ${ }^{1}$ Suzhou Institute of Biomedical Engineering and Technology, Chinese Academy of Science, Suzhou, China \\ ${ }^{2}$ Jiangsu Key Laboratory of Medical Optics, Suzhou, China \\ Email: yangjieabc1988@163.com
}

How to cite this paper: Gu, H.D., Shao, Z.X., Yang, J., Zheng, C.Q. and Chen, R.T. (2017) Analysis and Optimum Design of a Portable Q-Switch Based on Raman-Nath Diffraction. Optics and Photonics Journal, 7, 20-25.

https://doi.org/10.4236/opj.2017.78B004

Received: April 26, 2017

Accepted: August 7, 2017

Published: August 10, 2017

\begin{abstract}
The optimal acousto-optic interaction length in Raman-Nath diffraction is investigated by using deliberately designed apparatus working with pure water. Then the optimum interaction length, the suitable dimensions of the transducer, and the required index difference dominated by the ultrasonic frequency and power for achieving the ultimate efficiency are analyzed. The portable device analogy to the solid appliance is designed based on the optimization. Taking advantage of the device the highest diffraction efficiency of more than $98 \%$ is obtained. The feasibility of using the portable device to act as a Q-switch for ultraviolet and visible lasers is discussed.
\end{abstract}

\section{Keywords}

Raman-Nath Diffraction, Optimum Interaction Length, Ultimate Diffraction Efficiency

\section{Introduction}

The features of Raman-Nath (R-N) acousto-optic diffraction are with short interaction length and multi-order diffraction. The distribution of the energy in each diffractive order follows Bessel function [1]. It is significant that the zero order term, $J_{0}(\varphi)$, of Bessel function is equal to zero while the synthetics $\varphi$ takes the values of $2.405,5.520 \ldots$. This manifests that the zero order diffraction (the incidence passed the acousto-optic medium) would become a dark field and all the energy is diffracted into all the other orders. In this case the total diffractive efficiency attains to $100 \%$. However, so far, such a high efficiency has not been reported. Recent years most of researchers pay their attentions to Braggs' devices and their applications, which has a sole diffractive beam (e.g. [2] [3] [4]). And only a few of them studies to R-N diffraction [5] because its energy disperses in- 
to the multi-order diffractions. However, if considering the zero order can vanish at the ultimate efficiency, it predicts that taking advantage of the zero order diffraction and the short interaction length of $\mathrm{R}-\mathrm{N}$ regime a portable Q-switch device is feasible at the ultimate efficiency.

In this paper the apparatus working with pure water for studying the interaction length is designed first. The optimum interaction length and the dimensions of the transducer against the ultrasonic for achieving the ultimate efficiency are analyzed. Based on the analyses, the portable device analogy to the solid appliance is designed. The highest diffractive efficiency of more than $98 \%$ is achieved with the portable device. Finally, the feasibility of the portable device acting as a $\mathrm{Q}$-switch for ultraviolet and visible lasers is discussed.

\section{Experimental Apparatus}

Without putting the sheet of foam, or the medium is driven by standing sound wave. The multi-order diffraction is occurred as Figure 1 .

A glass tank sized $80 \times 60 \times 60 \mathrm{~mm}$ and filled of pure water in depth of $2 / 3$ the tank is taken as the acousto optical cell. The transducer-head with heat radiator sat on an adjustable mechanism which can be slide along the trail mounted on the upper frame of the water tank, see Figure 1. The head is inserted uprightly into the water. The transducer sized $20 \times 10 \times 0.2 \mathrm{~mm}^{3}$, which is driven by the homemade ultrasonic generator. (The largest acoustic power to be fed into the transducer is about 5 watts) The ration of standing wave is less than 1:1.2. A He-Ne laser $(632.8 \mathrm{~nm})$ and a CW Nd: YAG/KTP laser $(532 \mathrm{~nm})$ are employed as the light sources. The incidence is adjusted to be $3-5 \mathrm{~mm}$ beneath the transducer.

To measure the interaction length between the sound and the light, an aluminum block with a hole of $\varphi_{3} \mathrm{~mm}$ is deliberately put in the sound field and beneath the transducer-head to cut off the field. The diffracted beam can run through the hole and is received by the photo diode. The initial position of the fore-rim of the transducer, $P_{0}$, aims exactly to the fore-surface of the aluminum block. In this case the laser is shielded from the sound field by the aluminum block. Starting from $P_{0}$ the head is drawn to slide forward against the block so that the laser could enter the sound field. Through carefully adjusting the orientation of the transducer to make it nearly normal incidence, it is found that no

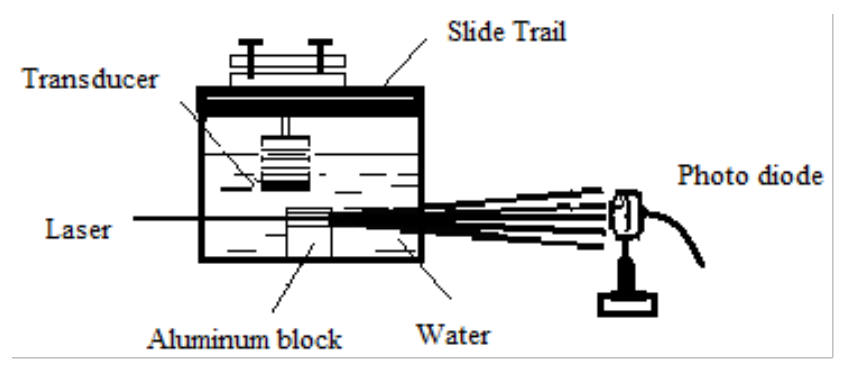

Figure 1. The apparatus for measuring the interaction length in $\mathrm{R}-\mathrm{N}$ diffraction. 
sooner the laser enter the sound field than the diffraction generated. While the head is drawn step be step the intensity of the diffracted beam was recorded. Then the diffractive intensity as a function of the interaction length is obtained. For avoiding disturbance from the sound a thin layer of porous material is covered on the aluminum block to absorb the sound.

\section{Results Analysis}

When applying the sound wave with the frequency of $\mathrm{f}_{\mathrm{s}} \sim 10 \mathrm{MHz}$ and the power of $\sim 1 \mathrm{~W}$, the diffractive efficiency, up to the third order, as the function of the interaction length are measured. Here, the data of the first order is taken and listed in Figure 2. It is apparently that the transverse coordinates, or the interaction length, of all the peaks of the curves, are almost at the same scale of $\sim 8 \mathrm{~mm}$. This shows that the optimum interaction length is $\sim 8 \mathrm{~mm}$ in this case.

In terms of the experimental results, the portable Q-switch working with pure water is designed as the photo in Figure 3. The body of the switch is made of aluminum with the dimensions of $40 \times 40 \times 17 \mathrm{~mm}^{3}$. The transducer volume is 8 $\times 0.2 \mathrm{~mm}^{3}$. The ration of standing wave for the electric network to feed the supersonic is practically designed to the less than $1: 1.2$.

In order to observe the process evolving toward the ultimate efficiency, applying the supersonic with frequency of $\sim 10 \mathrm{MHz}$ to the portable device, the

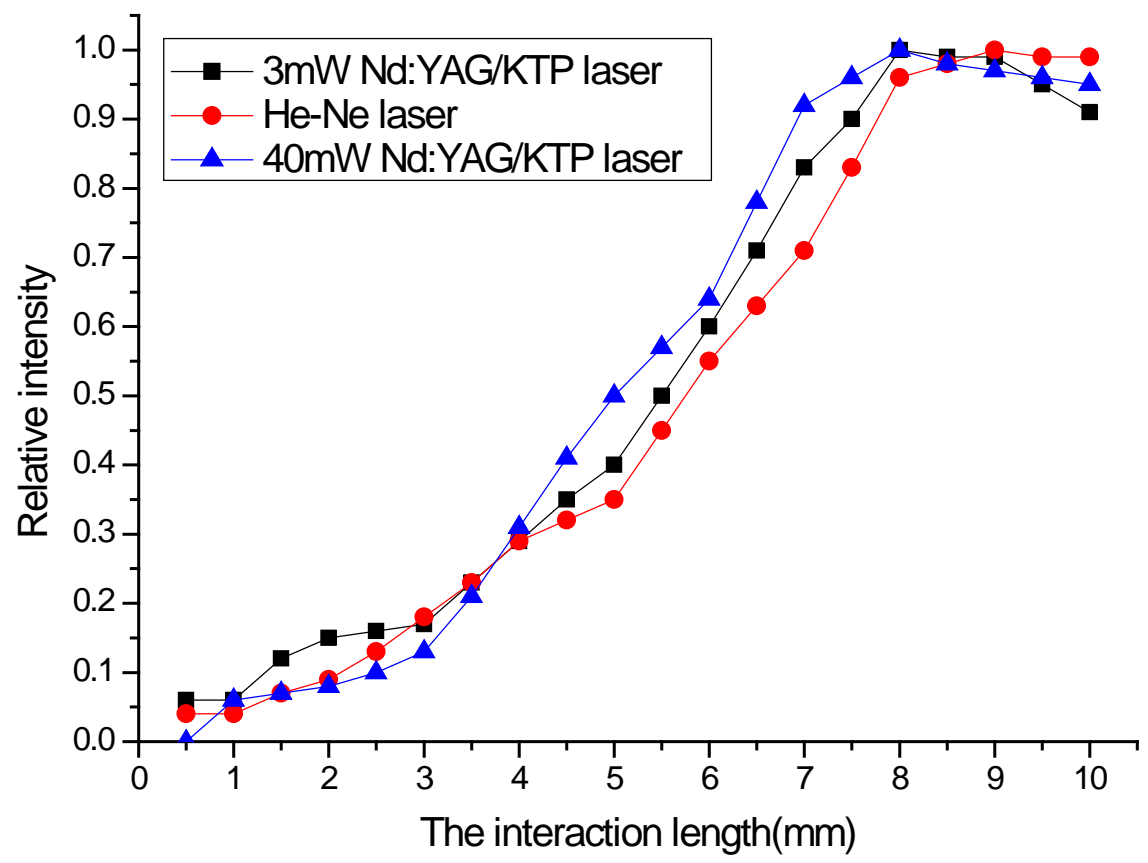

Figure 2. The data are the relative intensity of the first order diffraction versus the position of the prism at the sound frequency of $\sim 10 \mathrm{MHz}$ by using the lasers with different wavelengths and different power. The curve marked with the squares illustrates the data for the green Nd: YAG/KTP laser with the power of $3 \mathrm{~mW}$. The curve with the circles is the data for the He-Ne laser. The curve with the triangles is the data for the Nd: YAG/KTP laser with $40 \mathrm{~mW}$. 


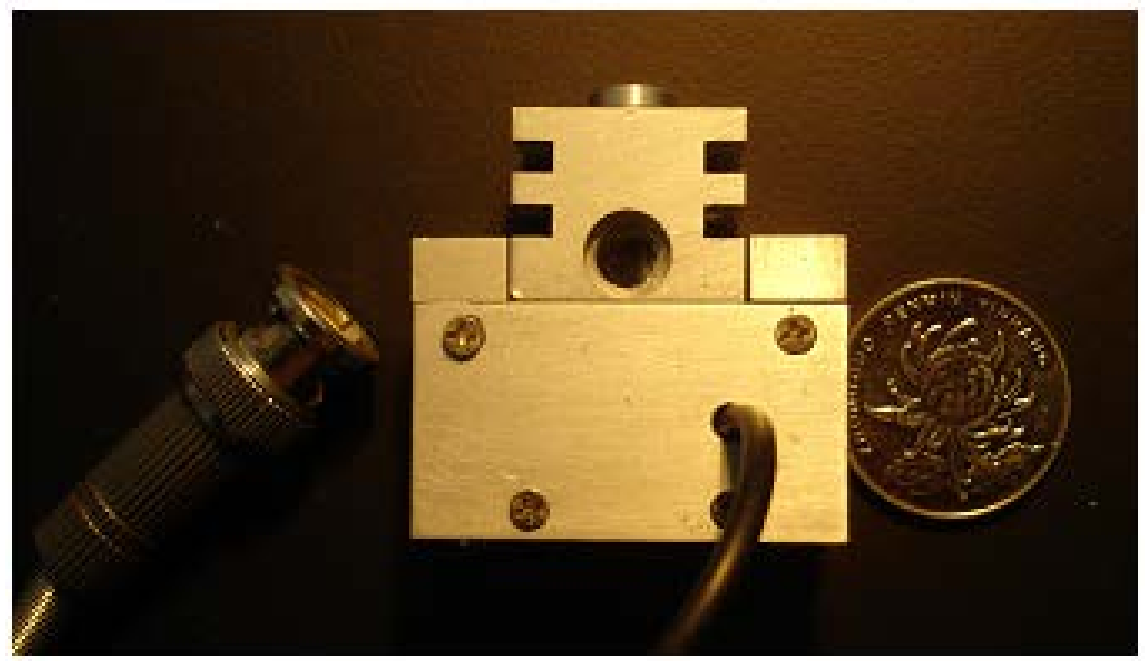

Figure 3. The portable acousto-optic Q-switch device working with pure water.

patterns of the diffractive spots with a He-Ne laser at different supersonic power are photographed as Figure 4. The diffractive angle is measured as $\theta=3.1 \mathrm{mrad}$.

Bessel terms, $J_{m}(\varphi)$, represent the diffraction efficiency of mth order. Here, the acousto-optic synthesis, or the phase of the diffractive wave [1],

$$
\varphi=\left(2 n \pi / \lambda_{0}\right) \times \Delta n \times L
$$

where $\lambda_{0}$ is the wavelength of the incidence. $n$ is the index. $L$ is the interaction length. And $\Delta n$ is the index difference, which can be expressed by

$$
\Delta n=n^{3} \times P \times\left(2 \pi / \lambda_{s}\right) \times A
$$

With $P$ is the photoelastic coefficient. $\lambda_{s}$ is the supersonic wavelength. A is the amplitude of the medium driven by the sound.

Figure 4(a) is shot at the power of $1 \mathrm{~W}$. In this case the device is working at a little bit warmer than the room temperature. The efficiency of the +1 th or -1 th is measured as $27 \%$. Converting it into Bessel term, corresponding to $J_{1}(\varphi)=$ 0.52 for $\varphi=1.25$. Then $J_{0}(\varphi)=0.65$, or the efficiency of the zero-order is $\left[J_{0}(\varphi)\right]^{2}$ $=42 \%$. Indeed the energy in the zero spot is tested as about 0.4 of the incidence.

Figure $4(\mathrm{~b})$ is at $2.5 \mathrm{~W}$, which amplitude should be 1.58 times of case (a). Taking advantage of Equations (1) and (2), $\varphi=1.25 \times 1.58=1.98$, then, the efficiency of the first order is $\left[J_{1}(\varphi)\right]^{2}=33.4 \%$. The zero-order is $\left[J_{0}(\varphi)\right]^{2}=5.8 \%$. In this case the first order almost approaches to the maximum as the theory predicts. The zero-order turns to dark.

As the power is increased to $3.4 \mathrm{~W}$, for Figure $4(\mathrm{c}), \varphi=1.17 \times 1.98=2.32$. In this case the energy remains in the zero-order is only $0.3 \%$ of the incidence. Indeed, the zero-order almost vanishes on inspecting the photo. Taking $\lambda_{0}=633$ $\mathrm{nm}$ and $\mathrm{L} \sim 8 \mathrm{~mm}$, from Equation (1), $\Delta \mathrm{n}=0.22 \times 10^{-4}$. Appearently, even though the efficiency approaches to the ultimate, $\Delta \mathrm{n}$ is much less than the prediction of $10^{-4}$ [1]. In this case the temperature of the device rises to about $50^{\circ} \mathrm{C}$ without cooling. But the diffraction is stable. However, if increasing the supersonic 
power furthermore, the diffraction will not be stable. In fact the power density on the transducer is about $6.8 \mathrm{~W} / \mathrm{cm}^{2}\left(3.4 \mathrm{~W} /\left[\left(\pi \times 0.4^{2}\right) \mathrm{cm}^{2}\right]\right)$ in this case. It is comparable to the Bragg products, which transducer's dimension is $5 \times 0.8 \mathrm{~cm}^{2}$ and is fed the power of $20-50 \mathrm{~W}$. To lessen the heat, the interaction length $\mathrm{L}$ can be extended. This results in enlarging the dimensions of the device. Reversely, if cutting $\mathrm{L}$ for miniaturing the device, the heat must be more serious.

To test the feasibility of the portable device working as the Q-switch, exerting $8 \mathrm{kHz}$ square wave, shown as Figure 5(a), to modulate the sound, the modulation of the zero-order diffraction with a He-Ne laser is monitored as Figure 5(b). The results show that the modulation of the light is synchronized to the one of the sound. The rise time is in the order of $\sim 3 \mu$ s shown as Figure 5(c). This is comparable to the current Q-switch products.

\section{Conclusions}

The acousto-optic interaction length in R-N diffraction is first investigated by using the specially designed apparatus. The optimal interaction length and dimension of the transducer as well as the index difference for achieving the ultimate efficiency are realized. The portable device looked like solid configuration is designed based on the optimization. Taking advantage of the portable device the ultimate efficiency of $98 \%$ is achieved. Finally, the followings as the conclusion:

1) The optimal acousto-optic interaction length for achieving the ultimate efficiency relies essentially on the sound and almost not relates to the light.

2) To achieve the ultimate efficiency with the portable device the dimension of the transducer must match not only the frequency also the power of the sound.

3) Under the ultimate efficiency, the zero-order diffraction in dark field intimates the feasibility of R-N diffraction Q-switch.

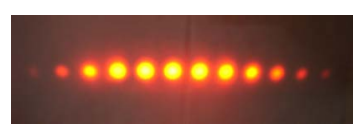

(a)

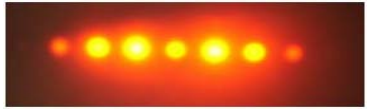

(b)

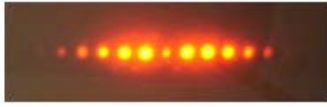

(c)

Figure 4. Photos of the pattern at different supersonic power. (a) At $\sim 1 \mathrm{~W}$; (b) At $\sim 2.5 \mathrm{~W}$; (c) At $\sim 3.4 \mathrm{~W}$.

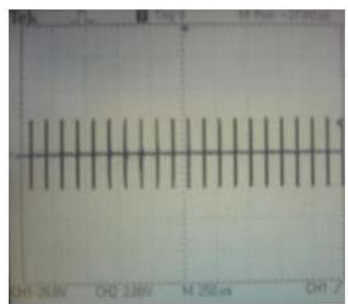

(a)

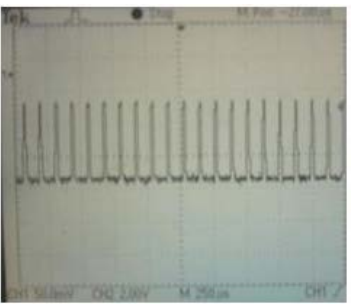

(b)

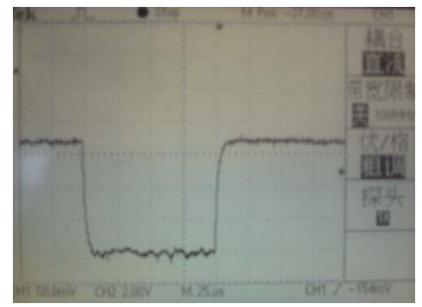

(c)

Figure 5. Photos shown the modulation signal and the switched laser analogy to Q-switch working. 


\section{Acknowledgements}

This work is funded by the Science and Technology Program of Jiangsu, China (grant No. BE2014641), the Science and Technology Program of Jinlin, China (grant No. 2015SYHZ0009), the Projects of International Cooperation MOST (grant No. 2015DFR10230) and the Program of Jiangsu Key Laboratory of Medical Optics (grant No. Y332021203-4).

\section{References}

[1] Ghatak, A.K. and Thyagarajan, K. (1989) Optical Electronics. Cambridge University Press, Cambridge. https://doi.org/10.1017/CBO9781139167857

[2] Jabczyński, J.K., Zendzian, W. and Kwiatkowski, J. (2006) Q-Switched ModeLocking with Acousto-Optic Modulator in a Diode Pumped Nd:YVO $\mathrm{V}_{4}$ Laser. Optics Express, 14, 2184-2190. https://doi.org/10.1364/OE.14.002184

[3] Zhu, H.Y., Zhang, G. and Huang, C.H. (2008) Diode-Side-Pumped Acousto-Optic Q-Switched 1319-nm Nd:YAG Laser. IEEE Journal of Quantum Electronics, 44, 480-484. https://doi.org/10.1109/JQE.2008.916698

[4] Yariv, A. (1989) Quantum Electronics. John Wiley \& Sons, Inc.

[5] Laude, V. (2003) General Solution of the Coupled-Wave Equation of Acousto-Optics. J. Opt. Soc Am. A, 20, 2307-2314. https://doi.org/10.1364/JOSAA.20.002307

Submit or recommend next manuscript to SCIRP and we will provide best service for you:

Accepting pre-submission inquiries through Email, Facebook, LinkedIn, Twitter, etc. A wide selection of journals (inclusive of 9 subjects, more than 200 journals)

Providing 24-hour high-quality service

User-friendly online submission system

Fair and swift peer-review system

Efficient typesetting and proofreading procedure

Display of the result of downloads and visits, as well as the number of cited articles

Maximum dissemination of your research work

Submit your manuscript at: http://papersubmission.scirp.org/

Or contact opj@scirp.org 